\title{
Atomic-resolution and Atomic-scale Imaging of Small Organic Molecules
}

\author{
PritiKharel, BlankaJanicek, Sang hyun Bae, Amanda Loutris and Pinshane Huang
}

University of Illinois at Urbana-Champaign, Urbana, Illinois, United States

The ability to image atomic arrangements in small organic molecules via transmission electron microscopy (TEM) offers a promising avenue for structural determination. Yet, attempts to image small-molecule crystals [1-4] or individual small molecules [5-8] at or even near atomic-resolution have had limited success because of their weak electron scattering and extreme beam sensitivity. $[9,10]$. For example, the critical dose of organic molecules typically ranges from $0.5-1000$ e-/Å2, several orders of magnitude lower than the doses required to image single carbon atoms in graphene [10]. As a result, developing new methods that can achieve high resolution at low dose is a key goal for enabling the study of small molecules and soft materials in the electron microscope. Here, we combine low-dose aberration-corrected annular dark-field scanning TEM (ADF-STEM) with advanced image processing methods from electron crystallography and single-particle analysis. We demonstrate that our approach can produce elementally sensitive images in atomically-thin 2D metallated porphyrin and phthalocyanine crystals with an effective resolution up to $1.3 \AA$ - sufficient to distinguish individual carbon and nitrogen atoms. To our knowledge, this is the highest resolution image ever reported of an individual small organic molecule using electron microscopy.

To begin, we apply low-dose ADF-STEM to three organic crystals- bilayer cobalt (II) (4-methoxyphenyl) porphyrin (CoTMPP), monolayer zinc phthalocyanine ( $\mathrm{ZnPc})$, and monolayer chlorinated copper phthalocyanine $(\mathrm{CuPcCl})$ on single-layer graphene support. Due to low-dose imaging constraints, the images of the molecules are dominated by a high level of noise. To extract the structural features from noisy frames, we locate, align and combine frames acquired from 102 - 105 individual molecules into a single averaged image, also referred to as a class average. As shown in Fig. 1a, our averages of CoTMPP show the orientation of methoxyphenyl groups, which appear as propeller-like features around the bright cobalt center. For $\mathrm{ZnPc}$ (Fig. 1b), we locate the metal center, visualize the 4-fold symmetry of the molecule, and even distinguish the carbon rings in the macrocycle, such as the benzene rings at the edge of the molecule. Our images of $\mathrm{CuPcCl}$ reveal the light elements in the connecting macrocycle as well as the structure of the four isoindole rings between the metal atom at the center and the chlorine atoms at the edge (Fig. 1c). We find the effective resolution of the class averages is $2.0 \AA$ for CoTMPP, $1.9 \AA$ for $\mathrm{ZnPc}$, and $1.3 \AA$ in $\mathrm{CuPcCl}$.

Importantly, we find that advanced data processing methods are key for atomic-resolution images. In Figure 2, we show final images generated using the same data, but differing class averaging methods: 1) thresholdbased averaging (Fig. 2a), 2) reference-based averaging (Fig. 2b), 3) iterative classification (Fig. 2c), and 4) multiple iterative classification (Fig. 2d). The first two simply locate, align, and average individual frames, while the latter two additionally remove frames of damaged, poorly centered, and misidentified molecules from the final average. By taking line profiles of the image intensity across the azimethine $\mathrm{C}-\mathrm{N}$ bond for each averaging method and comparing them to simulated data (Fig. 4f), we resolve individual carbon and nitrogen atoms only in the multiple iterative classification approach. This implies that the ability to isolate "good" frames is crucial for retaining atomic-resolution structural information of the molecules. Together, our results indicate a new route for atomic-resolution electron microscopy imaging to directly solve the structures of small organic molecules [11]. 


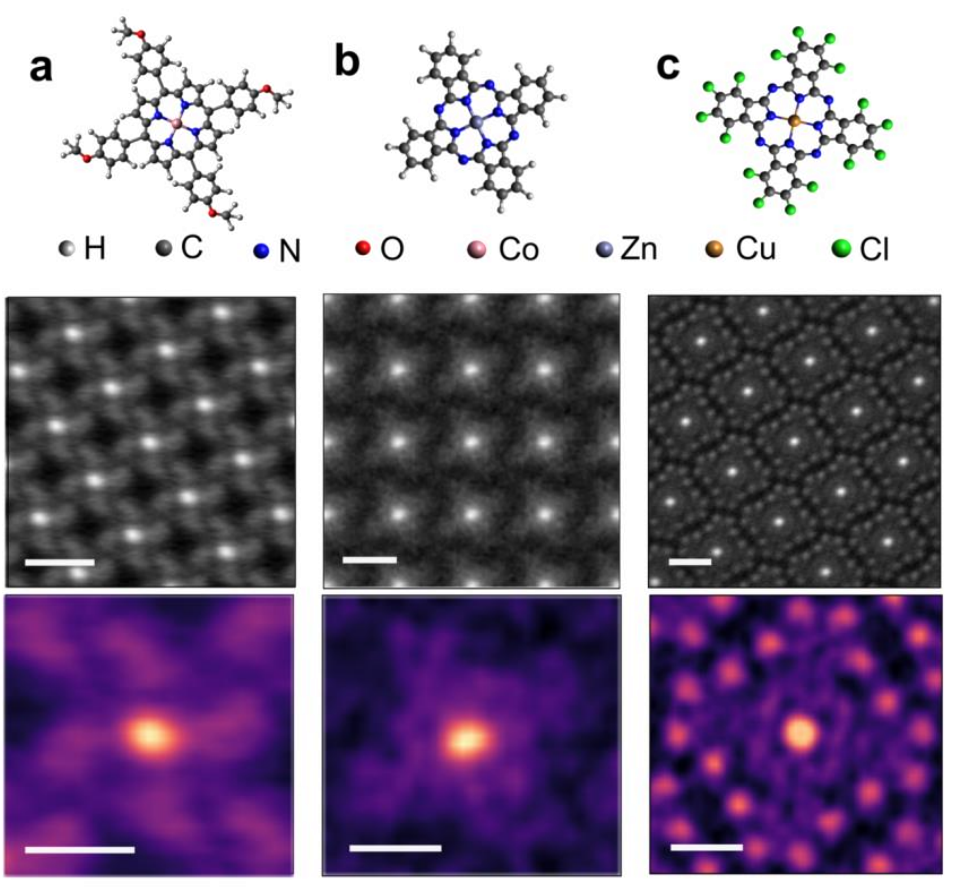

Figure 1. Atomic-scale and atomic-resolution imaging of small organic molecules using ADF-STEM. 2D structural representation (top) and experimental averaged images (middle and bottom) of (a) CoTMPP, (b) $\mathrm{ZnPc}$, and (c) $\mathrm{CuPcCl}$. The middle row of images shows the arrangements of molecules in each 2D crystal, and the bottom row shows a magnified, low pass filtered image of one molecule. Scale bars are $1 \mathrm{~nm}$ (middle) and $5 \AA$ (bottom).

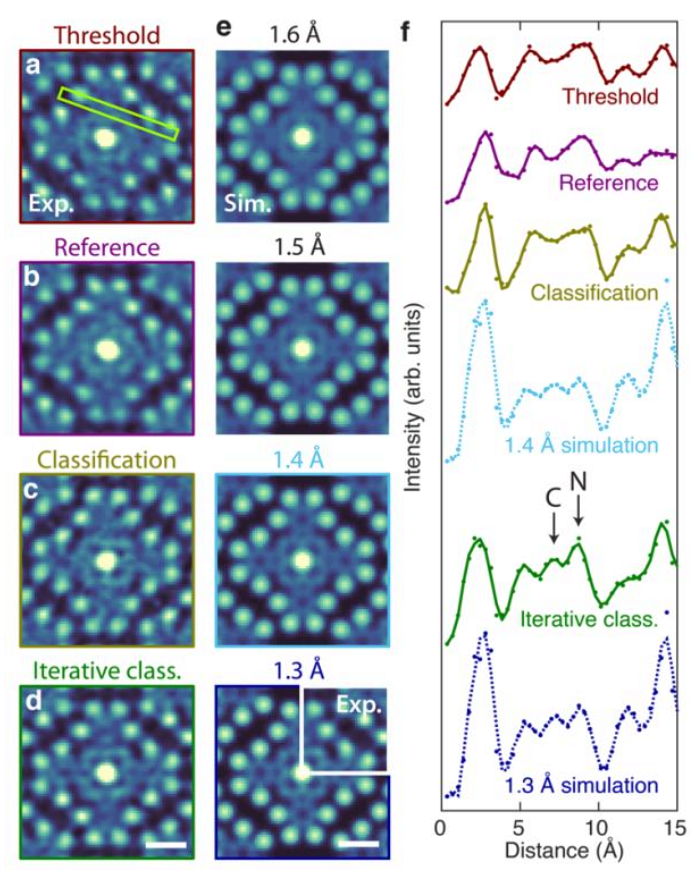

Figure 2. Data processing methods for atomic resolution imaging of monolayer $\mathrm{CuPcCl}$. (a-d) Experimental ADF-STEM data after processing using either (a) threshold-based averaging (1373 frames), (b) reference-based averaging (3924 frames), (c) a single round of classification (320 frames), and (d) 
iterative classification (140 frames). All images are low-pass filtered. (e) Multislice ADF-STEM simulations of monolayer $\mathrm{CuPcCl}$ with probe sizes (FWHM) from $1.3 \AA-1.6 \AA$. The upper right corner of the $1.3 \AA$ simulation is overlaid with a quadrant of the experimental image in D. (f) Line profiles across a $\mathrm{C}-\mathrm{N}$ bond, indicated by the green rectangle in (a). Data are shown as points, displayed with a smoothed (Savitsky-Golay filtered) line as a guide to the eye. In the iterative class average, intensity peaks corresponding to individual $\mathrm{C}$ and $\mathrm{N}$ atoms are visible, and the $\mathrm{N}$ appears brighter than $\mathrm{C}$. We estimate the resolution obtained using the iterative classification method is $1.3 \AA$. Scale bars are $5 \AA$.

\section{References}

[1] M Haruta et al., Mol. Cryst. Liq. Cryst. 492 (2008), p. 200.

[2] M Haruta et. al., Sci. Rep. 2 (2012).

[3] K Yoshida et al., Ultramicroscopy 159 (2015), p. 73.

[4] A Mittelberger et al., Sci. Rep. 8 (2018).

[5] M Koshino et al., Science 316 (2007), p. 853.

[6] K Cao et al., Sci. Adv. 6 (2020).

[7] A Markevich et al., Nanoscale 8 (2016), p. 2711.

[8] K Harano et al., J. Am. Chem. Soc. 136 (2014), p. 466.

[9] R Hovden et al., Ultramicroscopy 123 (2012), p. 59.

[10] R Egerton Micron 119 (2019), p. 72.

[11] This work was supported by the Packard Foundation and an NSF CAREER award DMR-1846206. The experimental work was carried out in the Materials Research Laboratory and utilized facilities provided by NSF-MRSEC award number DMR-1720633. 